\title{
Radar Cross-Section Study of Cylindrical Cavity-Backed Apertures with Outer or Inner Material Coating: The Case of H-Polarization
}

\author{
Dilek Çolak, Student Member, IEEE, Alexander I. Nosich, Member, IEEE, and Ayhan Altıntaş, Senior Member, IEEE
}

\begin{abstract}
A dual-series-based solution is obtained for the scattering of an $\mathbf{H}$-polarized plane wave from a slitted infinite circular cylinder coated with absorbing material from inside or outside. For both cases, numerical results are presented for the radar cross section and comparisons are given for two different realistic absorbing materials. The radar cross-section dependencies are also given for the aspect angle of the scatterer and the thickness of the absorbing layer.
\end{abstract}

\section{INTRODUCTION}

T HE motivations for an extensive study of electromagnetic wave scattering from partially open two-dimensional (2D) and three-dimensional (3-D) cavities are well known. Indeed, cavity-backed apertures (CBA) like cockpits and air inlets contribute a great deal to the radar cross section (RCS) of a jet aircraft.

Such cavities are also noted for the set of internal resonances which can be used, in principle, for recognizing a target. Actually, it is a similar phenomenon which makes it possible to detect and discriminate between different kinds of fish when searching for the tuna in deep-sea fishing. The sonar echo signals display a resonant response at the frequency depending on the size and density of the air bag of a fish. So, to avoid the detection and identification, besides keeping the RCS as low as possible, the internal resonances are needed to be suppressed. For these reasons, the walls of the cavity usually are loaded, i.e., coated with some lossy material.

The CBA problems including open-ended waveguides and slitted cylindrical shells have been treated by various analytical [1] and [2], numerical [3]-[7] or approximate asymptotic approaches [8]-[13]. The dual-series-based Riemann-Hilbert Problem (RHP) approach is based on a combination of analytical and numerical techniques and, for certain canonical geometries, it ensures any desired accuracy within the digital precision of a computer [14]-[19]. In [19], we have applied the RHP technique to solve the problem of E-wave scattering from a CBA formed by a slitted infinite circular cylinder

Manuscript received August 10, 1993; revised October 24, 1994. This work was supported in part by the NATO-SFS, TU-MIMIC, the Scientific and Technical Research Council of 'Turkey (TUBITAK), and the Matsumae International Foundation.

D. Çolak was with the Department of Electrical and Electronics Engineering, Bilkent University, Bilkent, Ankara 06533 Turkey. She is now with ElectroScience Laboratory, Ohio State University, Columbus, OH 43212 USA. A. Altıntaş is with the Department of Electrical and Electronics Engineering, Bilkent University, Bilkent, Ankara 06533 Turkey.

A. I. Nosich is with the Institute of Radiophysics and Electronics, Ukranian Academy of Sciences, 310085, Kharkov, Ukraine. IEEE Log Number 9410738. with inner or outer material coating. The uncoated CBA like this (H-case) was studied in [16] with the emphasis to the surface current computations. The numerous Soviet papers on scattering from slitted cylinders (single and multiple ones) were cited in [14]. In this paper, the H-wave excitation for the same problem as [19] is considered. The derivations of basic equations are very similar to the E-case, so we shall omit the details, making the needed references to [19] and pointing out the differences. Instead, we shall concentrate on the physical analysis of numerical results for the RCS.

The paper is organized as follows. In Section II, we present the formulation of the problem. The dual-series equations are derived and then solved via RHP approach in Section III. Sample numerical results are presented in Section IV. Unlike the E-polarized case, there appears a low-frequency spike in the RCS which does not correspond to any interior resonance of the closed cavity. This resonance is called quasi-static or slot-mode, and we discuss its nature from physical and mathematical points of view. Main attention is paid to the resonant behavior of RCS and its suppression by means of absorbing coating. Finally, some conclusions are given in Section V. Throughout this paper the time dependence $e^{-i w t}$ has been assumed and suppressed.

\section{FORMULATION}

The geometries analyzed in this paper are shown in Fig. 1(a) and (b). The perfectly conducting screen with radius " $a$ " is taken to be in the interval of $\theta<\left|\varphi-\varphi_{0}\right| \leq \pi$. Angular width of the slit is $2 \theta$, and the angle between its center and the $x$ axis is $\varphi_{0}$. The cavity is coated with an absorbing material of thickness $t$ and the relative permittivity and permeability $\epsilon_{r}$ and $\mu_{r}$, respectively.

First, consider the inner coating shown in Fig. 1(a). The total field can be expressed via the single $H_{z}$ component as follows

$$
H_{z}(\vec{r})= \begin{cases}H_{z}^{s c}(\vec{r})+e^{i k_{0} x}, & r>a, \\ H_{z}^{s c}(\vec{r}), & b<r<a, \\ H_{z}^{s c}(\vec{r}), & r<b\end{cases}
$$

where $e^{i k_{0} x}$ is the incident plane wave of unit amplitude.

The problem formulation for the H-polarized incidence is similar to the E-polarized case in [19]. The scattered field $H_{z}^{s c}$ has to satisfy the 2-D Helmholtz equation, with coefficient $k_{0}^{2}=\omega^{2} \mu_{0} \epsilon_{0}$ or $k^{2}=k_{0}^{2} \epsilon_{r} \mu_{r}$, outside or inside the coating, respectively, where $k_{0}$ is the free-space wave number. The boundary conditions are of two kinds. First kind is the 


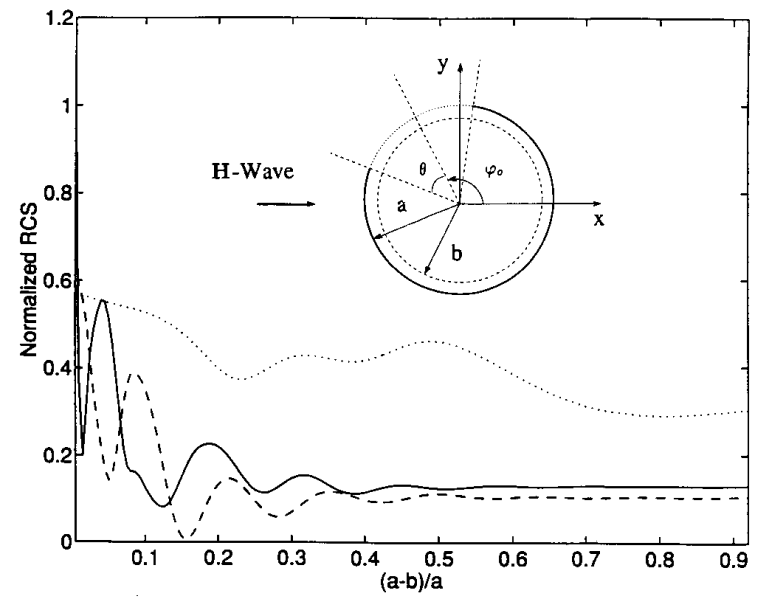

(a)

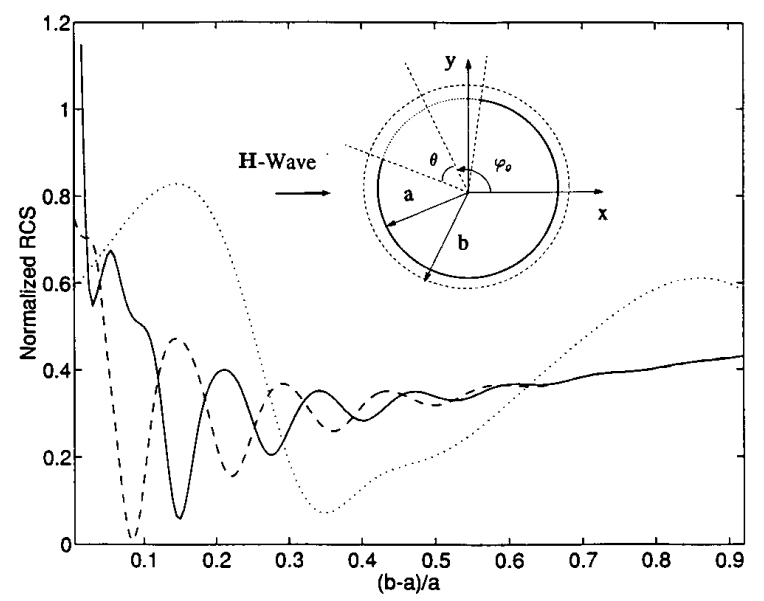

(b)

Fig. 1. (a) Inner-coated and (b) outer-coated CBA geometries, together with corresponding RCS dependencies on the relative thickness of lossy magnetic coating. Aperture size is $2 \theta=60$ degrees, orientation $\varphi_{0}=180$ degrees. Solid line: $k_{0} a=9.39$; dashed line: $k_{0} a=8.5$; dotted line: $k_{0} a=1.71$.

continuity of $E_{\varphi}$ on the closed contours $r=a$ as well as $r=b$, and the continuity of the $H_{z}$ on $r=b$. The second kind is mixed (dual-type) condition at $r=a$, the continuity of $H_{z}$ in the aperture region and vanishing $E_{\varphi}$ on the screen.

Because of the sharp edges of the screen, we specify the field behavior near the edges, in the form of the Meixner condition ensuring the local integrability of the field energy. Finally, the Sommerfeld radiation condition should be satisfied far from the scatterer.

\section{Derivation ANd Solution of DuAl SERIES EQuations}

The circular shape of the boundaries of the geometry in Fig. 1(a) invokes using the total field expansions in three regions as

$$
\begin{aligned}
H_{z}= & \sum_{n=-\infty}^{\infty}\left\{\begin{array}{cc}
A_{n} H_{n}\left(k_{0} r\right)+i^{n} J_{n}\left(k_{0} r\right), & r>a \\
B_{n} J_{n}(k r)+C_{n} H_{n}(k r), & b<r<a \\
D_{n} J_{n}\left(k_{0} r\right), & r<b
\end{array}\right\} \\
& \cdot e^{i n \varphi}
\end{aligned}
$$

where $J_{n}$ and $H_{n}$ are the Bessel and Hankel functions of first kind, respectively.

The number of coefficients in (2) can be reduced by applying boundary conditions that are valid on the closed contours, that is, the continuity of $E_{\varphi}$ at $r=a$ and $r=b$ as well as the continuity of $H_{z}$ at $r=b$. Hence we obtain

$$
\begin{aligned}
& B_{n}=Z_{r}^{-1} \frac{i^{n} J_{n}^{\prime}\left(k_{0} a\right)+H_{n}^{\prime}\left(k_{0} a\right) A_{n}}{J_{n}^{\prime}(k a) \xi_{n}-H_{n}^{\prime}(k a) \eta_{n}} \xi_{n}, \\
& C_{n}=-Z_{r}^{-1} \frac{i^{n} J_{n}^{\prime}\left(k_{0} a\right)+H_{n}^{\prime}\left(k_{0} a\right) A_{n}}{J_{n}^{\prime}(k a) \xi_{n}-H_{n}^{\prime}(k a) \eta_{n}} \eta_{n}
\end{aligned}
$$

and

$$
D_{n}=\frac{2 i}{\pi k b} \frac{i^{n} J_{n}^{\prime}\left(k_{0} a\right)+H_{n}^{\prime}\left(k_{0} a\right) A_{n}}{J_{n}^{\prime}(k a) \xi_{n}-H_{n}^{\prime}(k a) \eta_{n}}
$$

where

$$
\begin{aligned}
\xi_{n} & =Z_{r} H_{n}^{\prime}(k b) J_{n}\left(k_{0} b\right)-H_{n}(k b) J_{n}^{\prime}\left(k_{0} b\right), \\
\eta_{n} & =Z_{r} J_{n}^{\prime}(k b) J_{n}\left(k_{0} b\right)-J_{n}(k b) J_{n}^{\prime}\left(k_{0} b\right), \\
Z_{r} & =\sqrt{\frac{\mu_{r}}{\epsilon_{r}}}
\end{aligned}
$$

and prime denotes derivative with respect to the argument.

The zero value of $E_{\varphi}$ on the screen and the continuity of $H_{z}$ in the aperture lead to dual-series equations for the expansion coefficients

$$
\begin{gathered}
\sum_{n=-\infty}^{\infty} x_{n} \gamma_{n}^{-1} e^{i n \varphi}=\sum_{n=-\infty}^{\infty} d_{n} e^{i n \varphi}\left|\varphi-\varphi_{o}\right|<\theta \\
\sum_{n=-\infty}^{\infty} x_{n} e^{i n \varphi}=0, \quad \theta<\left|\varphi-\varphi_{o}\right| \leq \pi
\end{gathered}
$$

where

$$
\begin{aligned}
x_{n} & =A_{n} H_{n}^{\prime}\left(k_{0} a\right)+i^{n} J_{n}^{\prime}\left(k_{0} a\right), \\
d_{n} & =2 i^{n+1}\left[\pi k_{0} a H_{n}^{\prime}\left(k_{0} a\right)\right]^{-1}
\end{aligned}
$$

and

$$
\gamma_{n}=\left[\frac{H_{n}\left(k_{0} a\right)}{H_{n}^{\prime}\left(k_{0} a\right)}-\frac{1}{Z_{r}} \frac{J_{n}(k a) \xi_{n}-H_{n}(k a) \eta_{n}}{J_{n}^{\prime}(k a) \xi_{n}-H_{n}^{\prime}(k a) \eta_{n}}\right]^{-1} .
$$

The asymptotic behavior of $\gamma_{n}$ as $|n| \rightarrow \infty$ can be studied based on the corresponding expressions for cylindrical functions [20], which yields

$$
\begin{gathered}
\gamma_{n} \sim-\frac{|n|}{k_{0} a}\left(1+\epsilon_{r} / \beta_{n}\right)^{-1} \\
\beta_{n}=1+2 \frac{\epsilon_{r}-1}{\epsilon_{r}+1}\left(\frac{b}{a}\right)^{2|n|} \\
\cdot\left[1-\frac{\epsilon_{r}-1}{\epsilon_{r}+1}\left(\frac{b}{a}\right)^{2|n|}\right]^{-1} .
\end{gathered}
$$

To reduce the problem into canonical dual-series equations, we make the following transformation

$$
y_{n}=x_{n} \gamma_{n}^{-1}+d_{n}
$$


and use the asymptotic behavior of $\gamma_{n}$ in (1) to obtain the following dual-series equations analogous to (11) and (17) of [19]

$$
\begin{aligned}
\sum_{n=-\infty}^{\infty} y_{n} e^{i n \varphi}=0, \quad\left|\varphi-\varphi_{o}\right|<\theta \\
\sum_{n=-\infty}^{\infty} y_{n}|n| e^{i n \varphi}=\sum_{\substack{n=-\infty \\
\theta<\left|\varphi-\varphi_{o}\right| \leq \pi}}^{\infty}\left[y_{n} \Delta_{n}-k_{0} a\left(\epsilon_{r}+1\right) \gamma_{n} d_{n}\right] e^{i n \varphi}
\end{aligned}
$$

where

$$
\Delta_{n}=k_{0} a\left(\epsilon_{r}+1\right) \gamma_{n}+|n| .
$$

The set (17) and (18) form canonical dual-series equations which can be solved by converting it into the RHP [14]-[17]. Further, we follow [19] and use RHP solution as

$$
\mu_{m}=\sum_{n=-\infty}^{\infty} K_{m n} \mu_{n}+S_{m}, \quad m=0, \pm 1, \cdots
$$

where

$$
\begin{aligned}
\mu_{n}= & \frac{A_{n}}{J_{n}^{\prime}\left(k_{0} a\right)} \\
K_{m n}= & \frac{H_{n}^{\prime}\left(k_{0} a\right) J_{n}^{\prime}\left(k_{0} a\right)}{H_{m}^{\prime}\left(k_{0} a\right) J_{m}^{\prime}\left(k_{0} a\right)} \frac{\gamma_{m}}{\gamma_{n}} \Delta_{n} W_{m n} \\
S_{m}= & \frac{-i^{m}}{H_{m}^{\prime}\left(k_{0} a\right)}-\frac{2 i^{m+1}\left(\pi k_{0} a\right)^{-1} \gamma_{m}}{J_{m}^{\prime}\left(k_{o} a\right)\left[H_{m}^{\prime}\left(k_{o} a\right)\right]^{2}} \\
& +\frac{\gamma_{m}}{H_{m}^{\prime}\left(k_{0} a\right) J_{m}^{\prime}\left(k_{0} a\right)} \\
& \cdot \sum_{n=-\infty}^{\infty} i^{n}\left[\frac{J_{n}^{\prime}\left(k_{0} a\right) \Delta_{n}}{\gamma_{n}}+\frac{2 i}{\pi k_{0} a H_{n}^{\prime}\left(k_{0} a\right)}\right] \\
& \cdot W_{m n}
\end{aligned}
$$

and

$$
W_{m n}=e^{i(n-m) \varphi_{0}}(-1)^{m+n} T_{m n}(-\cos \theta) .
$$

In (24), $T_{m n}$ are related to the Legendre polynomials and their explicit expressions are given in [19]. The coefficients $W_{m n}$ contain all the information about the angular geometry of the screen and are bounded: $W_{m n}<C|m-n+1|^{-1}|m n|^{-(1 / 2)}$, uniformly for all $\theta$ and $\varphi_{0}$; the rest of $K_{m n}$ behaves as $O\left(k_{0}^{2} a^{2} /|n|\right)$.

We treat the outer covering problem (see Fig. 1(b)) in a similar way. In (2), $a$ 's are replaced with $b$ 's, and afterwards the same procedure is followed. The resultant infinite system of linear equations is formally the same as (20), however, now

$$
\begin{aligned}
K_{m n}= & \frac{J_{n}^{\prime}\left(k_{0} a\right)}{J_{m}^{\prime}\left(k_{0} a\right)} \frac{H_{n}^{\prime}(k a) \xi_{n}-J_{n}^{\prime}(k a) \eta_{n}}{H_{m}^{\prime}(k a) \xi_{m}-J_{m}^{\prime}(k a) \eta_{m}} \\
& \cdot \frac{\gamma_{m}}{\gamma_{n}} \Delta_{n} W_{m n}
\end{aligned}
$$

and the coefficients are

$$
\begin{aligned}
\xi_{n} & =J_{n}(k b) H_{n}^{\prime}\left(k_{0} b\right)-Z_{r} J_{n}^{\prime}(k b) H_{n}\left(k_{0} b\right) \\
\eta_{n} & =H_{n}(k b) H_{n}^{\prime}\left(k_{0} b\right)-Z_{r} H_{n}^{\prime}(k b) H_{n}\left(k_{0} b\right) \\
\Delta_{n} & =|n|-k_{0} a\left(1+\epsilon_{r}\right) \gamma_{n} \\
\gamma_{n} & =\left[\frac{J_{n}\left(k_{0} a\right)}{J_{n}^{\prime}\left(k_{0} a\right)}-\frac{1}{Z_{r}} \frac{H_{n}(k a) \xi_{n}-J_{n}(k a) \eta_{n}}{H_{n}^{\prime}(k a) \xi_{n}-J_{n}^{\prime}(k a) \eta_{n}}\right]^{-1} .
\end{aligned}
$$

The right-hand part vector in (20) is now given by

$$
\begin{aligned}
S_{m}= & -i^{m} g_{m}^{-1}\left[H_{m}^{\prime}(k a) s_{2 m}-J_{m}^{\prime}(k a) s_{4 m}\right] \\
& +8 i \gamma_{m}\left(\alpha g_{m}\right)^{-1}\left[H_{m}^{\prime}(k a) \xi_{m}-J_{m}^{\prime}(k a) \eta_{m}\right]^{-1} \\
& +\left(\frac{\gamma_{m}}{g_{m}}\right) \sum_{n=-\infty}^{\infty} i^{n}\left\{\left[H_{n}^{\prime}(k a) s_{2 n}-J_{n}^{\prime}(k a) s_{4 n}\right] \Delta_{n} \gamma_{n}^{-1}\right. \\
& \left.+8 i|n| \alpha^{-1}\left[H_{n}^{\prime}(k a) \xi_{n}-J_{n}^{\prime}(k a) \eta_{n}\right]^{-1}\right\} W_{m n}
\end{aligned}
$$

where

$$
\begin{aligned}
\alpha & =\pi^{3}\left(k_{0} a\right)\left(k_{0} b\right)^{2} \mu_{r} \epsilon_{r}, \\
g_{m} & =J_{m}^{\prime}\left(k_{0} a\right)\left[H_{m}^{\prime}(k a) \xi_{m}-J_{m}^{\prime}(k a) \eta_{m}\right], \\
s_{2 n} & =J_{n}(k b) J_{n}^{\prime}\left(k_{0} b\right)-Z_{r} J_{n}^{\prime}(k b) J_{n}\left(k_{0} b\right)
\end{aligned}
$$

and

$$
s_{4 n}=H_{n}(k b) J_{n}^{\prime}\left(k_{0} b\right)-Z_{r} H_{n}^{\prime}(k b) J_{n}\left(k_{0} b\right) .
$$

In operator notation, (20) can be written as a regularized Fredholm-type equation

$$
(I-K) \mu=S
$$

where $\mu=\left\{\mu_{n}\right\}_{n=-\infty}^{\infty}, S=\left\{S_{n}\right\}_{n=-\infty}^{\infty}, I$ is identity matrix and $K=\left\{K_{m n}\right\}_{m, n=-\infty}^{\infty}$. The solution of (35) can be approximated with any desired accuracy by means of the truncation of the matrix at $N_{t r}>k_{0} D$, where $D=$ $\max (a, b) \sqrt{\epsilon_{r} \mu_{r}}$. The sequence of approximate solutions is guaranteed to converge to exact solution for any $k_{0} a, b / a, \epsilon_{r}$, $\mu_{r}, \theta, \varphi_{0}$, as $N_{t r} \rightarrow \infty$ [14]. In practice, however, $N_{t r}$ is verified to be not so large to provide the accuracy of $0.1 \%$ in the RCS computations, one has to take $N_{t r}=k_{0} D+10$. The truncation error can be reduced by using the transformation $\bar{\mu}_{n}=\sqrt{1+|n|} \mu_{n}$ to symmetrize the matrix behavior at larger $|m|,|n|$. If such a modified (symmetrized) matrix is solved, $N_{t r}=k_{0} D+5$ equations is enough for the same accuracy.

This point is clarified by Fig. 2 which gives the truncation errors versus $N_{t r}$, for the surface current density, and the RCS of unloaded cavity, defined as follows

$$
\begin{aligned}
\delta_{\mu} & =\frac{\max \left|\mu_{n}^{N}-\mu_{n}^{N+1}\right|}{\max \left|\mu_{n}^{N}\right|}, \\
\delta_{R C S} & =\frac{\left|\sigma_{b s}^{N}-\sigma_{b s}^{N+1}\right|}{\sigma_{b s}^{N}} .
\end{aligned}
$$

Besides, the same figure presents the errors of the Optical Theorem [21] and the Reciprocity Theorem. On introducing the far-field pattern as

$$
h_{z}^{s c}(\varphi) \equiv \lim _{r \rightarrow \infty}(i \pi k r / 2)^{1 / 2} e^{-i k r} H_{z}^{s c}(r, \varphi)
$$

and total scattering cross section as

$$
\sigma_{s c} \equiv(2 / \pi k) \int_{0}^{2 \pi}\left|h_{z}^{s c}(\varphi)\right|^{2} d \varphi
$$

these errors are evaluated as follows

$$
\begin{aligned}
& \delta_{O T}=2 \frac{\left|k_{0} \sigma_{s c}^{N}+4 \operatorname{Re} h_{z}^{s c, N}(0)\right|}{k_{0} \sigma_{s c}^{N}-4 \operatorname{Re} h_{z}^{s c, N}(0)}, \\
& \delta_{R T}=2 \frac{\left|h_{z}^{s c, N}\left(0, \varphi_{0}\right)-h_{z}^{s c, N}\left(0, \pi-\varphi_{0}\right)\right|}{\left|h_{z}^{s c, N}\left(0, \varphi_{0}\right)+h_{z}^{s c, N}\left(0, \pi-\varphi_{0}\right)\right|} .
\end{aligned}
$$




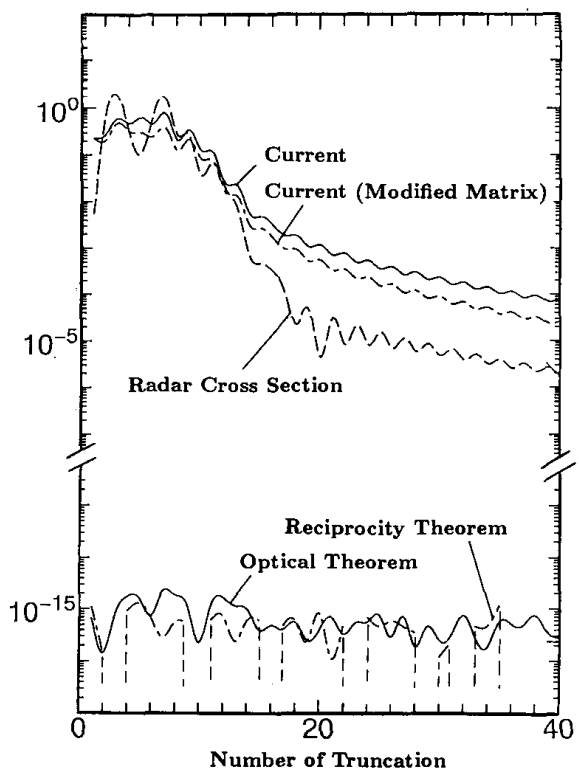

Fig. 2. Truncation errors versus the number of matrix truncation, for unloaded CBA characterized with $k_{0} a=10, \theta=90$ degrees, $\varphi_{0}=170$ degrees.

One can clearly see that the latter two quantities are always at the level of digital precision used, even at $N_{t r} \ll k_{0} D$, which points out to a term-by-term satisfying. Similar behavior is revealed for a loaded cavity taking into account the absorption inside the material layer. This test is enough to validate the computer code against the "bugs." It is worth mentioning that we have made same test for E-case [19] as well, and also checked the agreement between the results generated by our code and those of [18] and [3].

\section{NUMERICAL RESULTS AND DISCUSSION}

We studied numerically the RCS behavior of CBA in Fig. 1(a) and (b), using computer solution of matrix equations of the previous section and formula

$$
\begin{aligned}
\sigma_{b s} & \equiv \lim _{r \rightarrow \infty} 2 \pi r \frac{\left|H^{s c}(r, \pi)\right|^{2}}{\left|H^{i n}\right|^{2}} \\
& \approx \frac{4}{k_{0}}\left|\sum_{-N_{t r}}^{N_{t r}} \mu_{n} i^{n} J_{n}^{\prime}\left(k_{0} a\right)\right|^{2} .
\end{aligned}
$$

Normalization constant was taken as $\pi a$ which is the geometrical optics RCS value for the closed, perfectly conducting (uncoated) circular cylinder. The normalized RCS results are presented in Figs. 3-5 versus frequency for two different coating materials and three different orientations of the aperture. In all figures, the slit half-width, $\theta$, is taken as 30 degrees, and the materials used for coating are the same as in E-polarization case [19]. They are shellac, natural XL $\left(\epsilon_{r}=3.45+0.25 i, \mu_{r}=1\right)$ [22] (dashed curves), and poly2.5-dichlorostyrene $\left(\epsilon_{r}=7.3, \mu_{r}=0.91+0.32 i\right)$ [10] (solid curves), and we further refer to them as lossy electric and

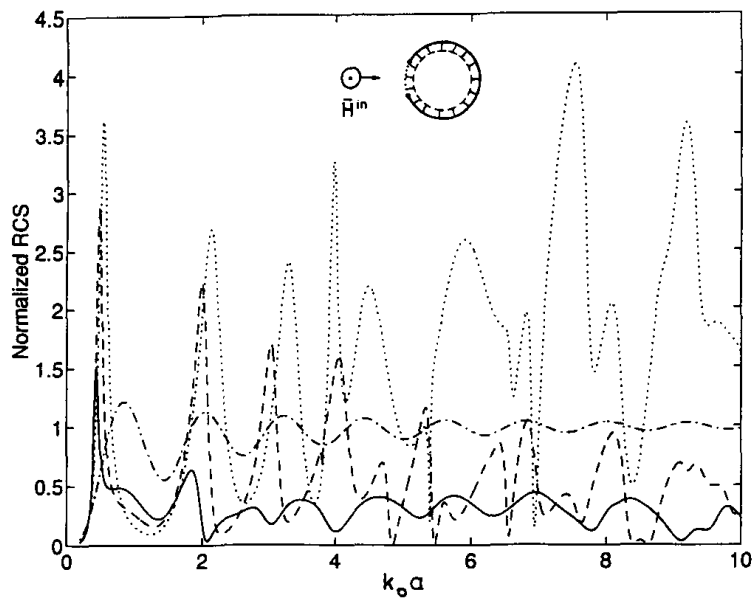

(a)

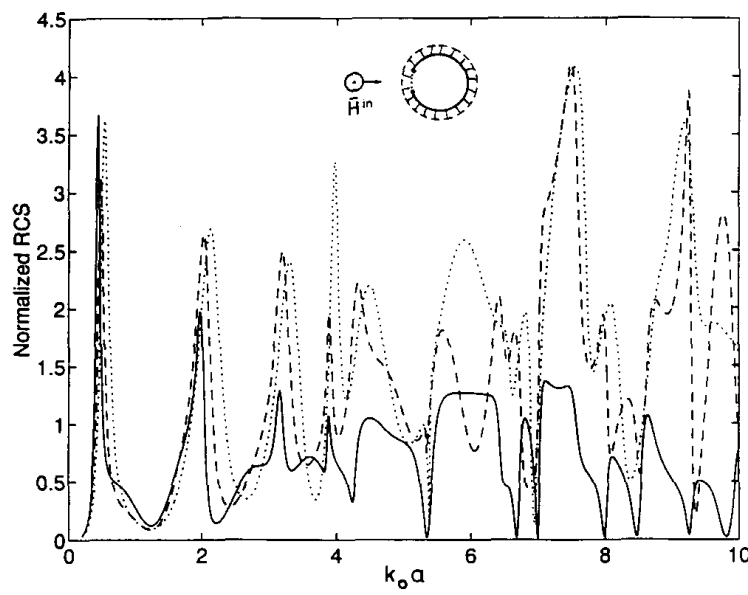

(b)

Fig. 3. The frequency scans of the normalized RCS of (a) inner-coated and (b) outer-coated CBA characterized with the aperture size $2 \theta=60$ degrees and symmetrical orientation, $\varphi_{0}=180$ degrees. Solid line: lossy magnetic coating; dashed line: lossy electric coating; dash-dotted line: closed uncoated circular cylinder; dotted line: uncoated CBA.

lossy magnetic coating. The thickness of the absorbing layer is $10 \%$ of the screen radius. For comparison, dotted curves represent the RCS calculated for the similar but uncoated CBA, and dash-dotted line in Fig. 3(a) gives RCS of a perfectly conducting circular cylinder. It is noted that for $\varphi_{0}=180$ degrees case, the average level of RCS of uncoated CBA is much higher than that of closed, uncoated circular cylinder of the same radius. In addition, strong resonances are observed as minima and maxima in the RCS. They are due to the excitation of the damped, natural modes of the screen as a CBA. Except for the first, low-frequency peak, all these modes originate from the eigenmodes of the closed-circular cavity, $H_{m n}$, being shifted in frequency and splitted, for $m \neq 0$, into even/odd pairs for which we keep the similar identifiers, $H_{m n}^{ \pm}$. To the best of our knowledge, it was in [23] where the low-frequency resonance has been first reported and explained in terms of an equivalent LC circuit (see also, [5], [6], and 


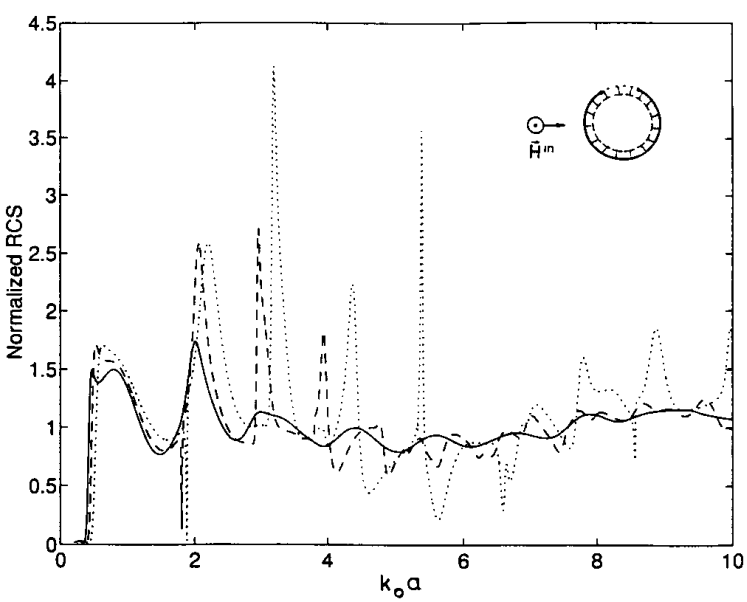

(a)

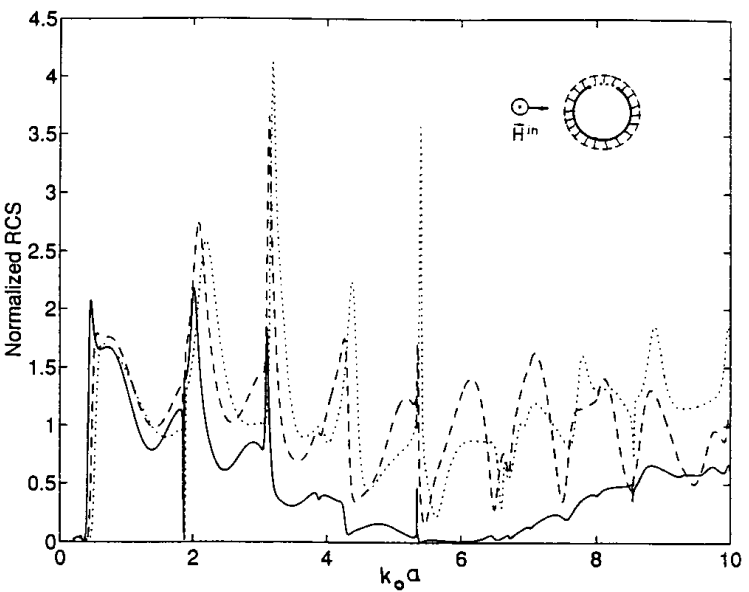

(b)

Fig. 4. The frequency scans of the normalized RCS of (a) inner-coated and (b) outer-coated CBA characterized with the aperture size $2 \theta=60$ degrees and nonsymmetrical orientation, $\varphi_{0}=90$ degrees. Solid line: lossy magnetic coating; dashed line: lossy electric coating; dotted line: uncoated CBA.

[18]). For a somewhat different scatterer, it was also explained in terms of equivalent ring resonator loaded by a opencircuit slit discontinuity (see [1]). The nature of this resonance is the same as that of so-called "slot mode" propagating with or without a leakage along a slitted waveguide [24] and [25].

From the mathematical viewpoint discussed first in [23], this low-frequency resonance has a singular nature. Indeed, any interior Neumann boundary-value problem is known to have the zero as the lowest eigenvalue with arbitrary constant as eigenfunction. In electromagnetics, however, this constant can be shown identically equal to zero. Therefore, strictly speaking, zero is not an eigenvalue. The effect of cutting the slot is to shift this zero pseudo-eigenvalue by a small complex number

$$
k_{00} a \approx\left(-2 \ln \sin \frac{\theta}{2}\right)^{-1 / 2}\left(1+\frac{i \pi}{16} \ln ^{-1} \sin \frac{\theta}{2}\right)
$$

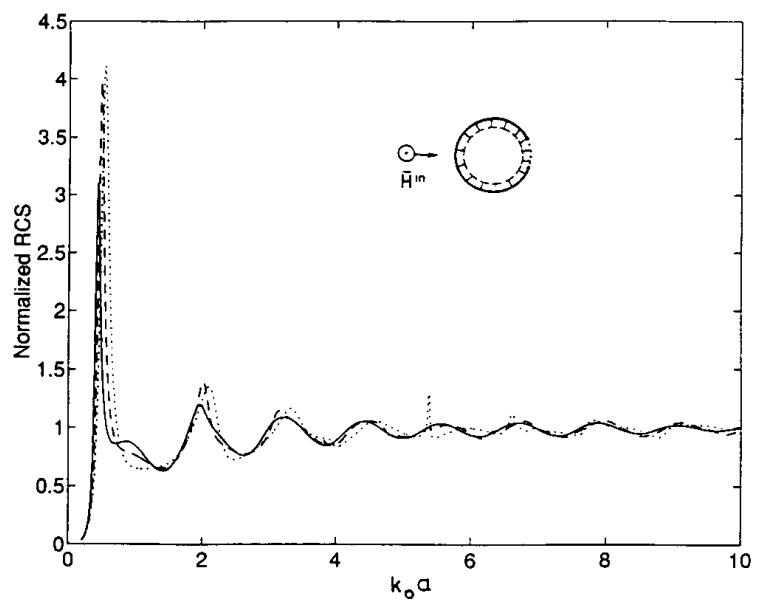

(a)

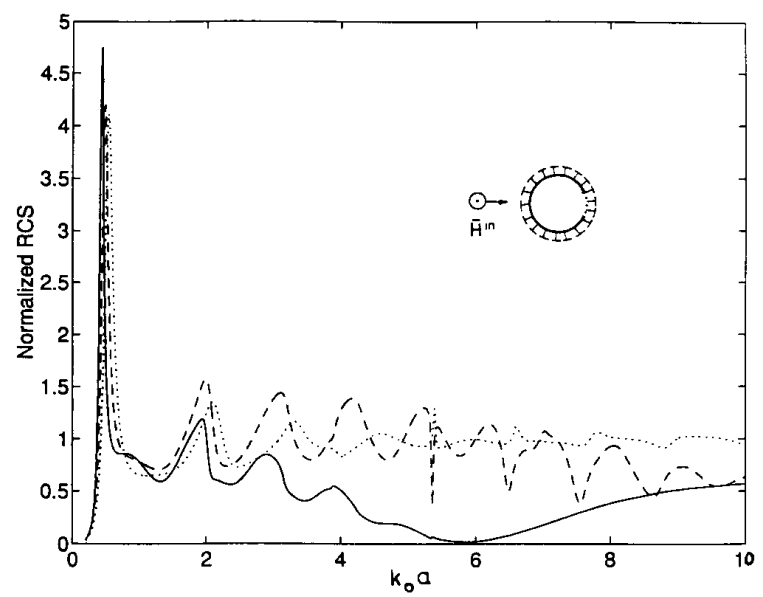

(b)

Fig. 5. The frequency scans of the normalized RCS of (a) inner-coated and (b) outer-coated CBA characterized with the aperture $2 \theta=60$ degrees located at the back side of the scatterer, $\varphi_{0}=0$. Solid line: lossy magnetic coating; dashed line: lossy electric coating; dotted line: uncoated CBA.

with a corresponding nonzero eigenfunction. As it was pointed out in [23], this low-frequency resonance is analogous to the so-called Helmholtz mode of oscillations in acoustic cavities investigated by Rayleigh [26]. We have denoted this lowfrequency resonance by $H_{00}$. The Helmholtz resonance is of special interest as at the resonant frequency, i.e., $k_{00}^{\prime}=\operatorname{Re} k_{00}$, the backscattering RCS is obtained asymptotically as

$$
\sigma_{b s}=\frac{4}{k_{00}^{\prime}}\left(1+8 k_{00}^{\prime 2} a^{2} \cos ^{4} \frac{\theta}{2} \cos ^{2} \phi_{0}\right)+O\left(k_{00}^{\prime 3} a^{4}\right)
$$

for an arbitrary-oriented-uncoated-slitted cylinder [15]. So, the resonant value of RCS tends to infinity as $\theta \rightarrow 0$, in sharp contrast with so-called Rayleigh rule for the low frequency H-scattering from smooth cylinders which is $\sigma_{b s}=O\left(k^{3} a^{4}\right)$.

For uncoated CBA, the shifted natural frequencies have been calculated previously in [15]. Under assumption that $\tau=\sin (\theta / 2) \rightarrow 0$, the matrix of (20) takes a quasi-diagonal 
form which enables one to carry out an iterative perturbation analysis of the characteristic equation $\operatorname{det}(I-K)=0$. The complex natural frequencies are found as asymptotic series

$$
\begin{gathered}
k_{m n}^{+} a \approx \nu_{m n}+\nu_{m n}\left[\delta_{m}\left(\nu_{m n}^{2}-m^{2}\right) \ln \tau\right]^{-1} \\
\cdot\left(1+i \zeta_{m n} \ln ^{-1} \tau\right)
\end{gathered}
$$

with $m=0,1,2, \cdots$ and

$$
k_{m n}^{-} a \approx \nu_{m n}+\nu_{m n} m^{2}\left(\nu_{m n}^{2}-m^{2}\right)^{-1} \tau^{2}\left(1-2 i \Upsilon_{m n} \tau^{2}\right)
$$

with $m=1,2, \cdots$, for the even and odd modes of the circular slitted cavity, respectively. Here

$$
\begin{gathered}
\zeta_{m n}=\pi^{-1} \nu_{m n}^{-2} \sum_{s=0, \neq m}^{\infty}\left|H_{s}^{\prime}\left(\nu_{m n}\right)\right|^{-2} \\
\Upsilon_{m n}=\pi^{-1} \nu_{m n}^{-2} \sum_{s=1, \neq m}^{\infty} s^{2}\left|H_{s}^{\prime}\left(\nu_{m n}\right)\right|^{-2}
\end{gathered}
$$

and $\delta_{0}=1, \delta_{s}=2$ for $s \neq 0$, while $\nu_{m n}$ is the $n$th zero of $J_{m}^{\prime}(x)$. The quantities of (47) and (48) are, except of a constant factor, the radiation power of a magnetic-line source or a pair of opposite-phase line sources, respectively, located on the surface of the cylinder (see [15]).

Note that for the symmetrical position of the slitted cylinder, i.e., when $\varphi_{0}=0$ degrees or 180 degrees, there may be excited only even modes, i.e., $H_{0 n}, H_{m n}^{+}$, however, for nonsymmetrical orientations both resonances, even and odd modes, i.e., $H_{m n}^{+}$and $H_{m n}^{-}$, do appear in closely spaced pair. All the peaks of RCS are of finite amplitude. This is because the perturbed eigen frequencies are now complex numbers with negative imaginary parts, (see (22), (24), and (25)). The Q-factors of odd modes are much higher than even modes since the imaginary part of $k_{m n}^{-}$is less than of $k_{m n}^{+}$as $\theta^{2} \ll$ $-\ln ^{-1} \theta$. The excited modes corresponding to the first five resonance frequencies for $\varphi_{0}=180$ degrees case are known as $H_{00}, H_{11}^{+}, H_{21}^{+}, H_{01}, H_{31}^{+}$. For an uncoated CBA with $\varphi_{0}=$ 180 degrees, the first several maxima of RCS in the numerical results show a good agreement with the real parts of the shifted natural frequencies (43), (45), and (46). Higher-order resonances, however, especially for nonsymmetric orientation of CBA, may develop themselves as minima as well. Contrary to E-polarized case, all the resonances are slightly higher than the corresponding closed-cavity eigenvalues. This is due to the difference in the direction of the induced current which flows circumferentially in the $\mathrm{H}$-polarization whereas axially in E-polarized case.

Fig. 3(a) and (b) demonstrate the effect of the absorbing material on the inner and outer wall of CBA, respectively, for the case of aperture in the illuminated region. Unlike the E-polarized case, a lossy electric coating is effective for reducing the lowest-order resonance peak, since in this case the electric field is not zero near the screen. As observed in these figures, when the frequency increases, the resonance peaks are reduced even more effectively. It is due to the fact that the field is concentrated near the walls of the cavity (the effect of the whispering gallery). Therefore, resonances of higherorder modes can be suppressed by coating the screen with the absorbing material from inside. Coating from outside has no

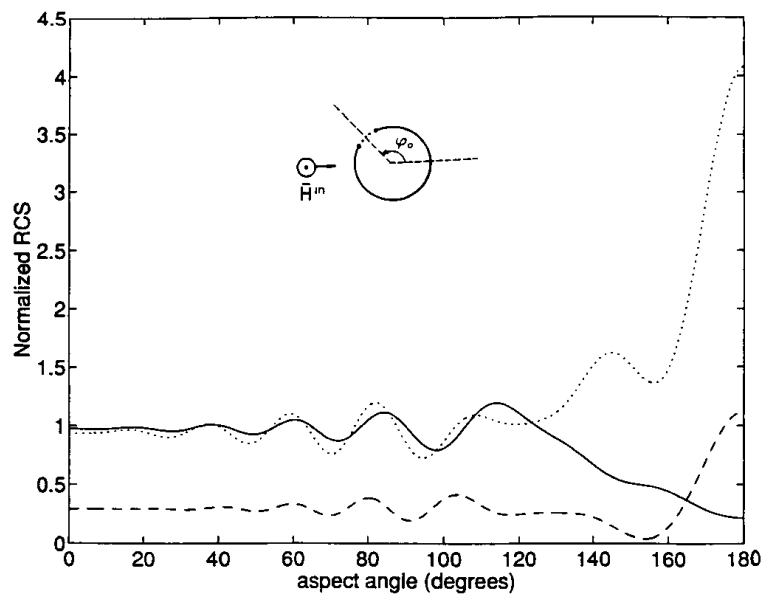

Fig. 6. The normalized RCS versus the orientation angle $\varphi_{0}$ of the aperture of CBA coated with lossy magnetic material, at $k_{0} a=7.54$. Solid line: inner-coated CBA; dashed line: outer-coated CBA; dotted line: closed uncoated circular cylinder.

effect on the Q-factor of the internal resonances but it helps to decrease the amplitude of the incident field entering the cavity. So, the average level of RCS is reduced as seen in Fig. 3(b), but the resonances are just shifted in frequency remaining still sharp which may cause the target to be identified.

An example of nonsymmetrical excitation is given by the case of 90-degree orientation, i.e., when the aperture is looking up. As seen in Fig. 4(a), coating from inside is again effective for suppressing the resonances. The resonance phenomena are greatly reduced if the frequency is increased, and especially if the magnetic coating is used. On the other hand, coating from outside is effective for reducing the average level of the RCS. But, there are still sharp resonances (see Fig. 4(b)), although with reduced amplitudes.

In Fig. 5(a) and (b), similar results are obtained for the case when the aperture is in the shadow region. We include these plots because, contrary to E-polarized case, there is a noticeable difference with the closed cylinder for $\mathrm{H}$-polarization (see Fig. 3(a), dash-dotted curve). This is due to fact that the E-polarized excitation induces only longitudinal current on a cylindrical scatterer, hardly reaching the shadow part of surface and hence not exciting the interior of CBA, whereas $H$-polarized excitation induces current in the circumferential direction. Therefore, the presence of aperture causes a stronger response than E-polarized case, especially at lower frequencies when there is no actual shadow zone on the surface of the CBA. That is why the first few resonances are equally effective for any aspect angle of the aperture. At higher frequencies, it is noted that the solid curve in Fig. 5(b) is very similar to the one in Fig. 4(b). The frequency value at which RCS has a broad minimum in those figures corresponds to minimum of the reflection coefficient for a lossy material slab backed by a perfectly conducting plane.

The normalized RCS dependencies on the aperture orientation angle $\varphi_{0}$ are presented in Fig. 6 for a sample frequency. Only lossy magnetic-coating results are given. One notes again 
that at on-aperture incidence, the inner coating serves much better for reducing the RCS, than the outer coating.

For the dependence of RCS on the thickness of the absorbing layer, let us return to Fig. 1(a) and (b). The results are obtained only for the lossy magnetic material at three sample frequencies. General behavior of RCS is very similar to E-polarization [19]. The curves have several oscillations at first, due to the interference of the waves reflected from the both surfaces of material "window" at the aperture. As the thickness is increased, for the inner loading (Fig. 1(a)) they stabilize at a low level after crossing the internal caustic, due to the absorption of the whispering-gallery-shaped field in the coating. For the outer loading (Fig. 1(b)), they keep rising without oscillations as the outer field has no circumferential caustic. The location of the minima in Fig. 1(a) and (b) are in good agreement with the calculated ones for a lossy material slab, especially for higher frequencies when our circularly curved layer is closer to a planar slab. Hence, for practical purposes, the thickness of the "window" can be chosen so that at front incidence RCS has a minimum value at a specified frequency (cockpit model). On the other hand, if the absorbing layer were covering only the walls of CBA (air inlet model), but not the aperture itself, the interference oscillations should be absent.

\section{CONCLUSIONS}

The problem of a time-harmonic plane $\mathrm{H}$-wave scattering from a thin, perfectly conducting, slitted-infinite cylinder has been analyzed. The slitted cylinder is coated with absorbing material either from inside or from outside. Our aim was to study the effect of coating on the resonances which appear in RCS signatures of cavities. We first reduced the problem into dual series equations and then applied RHP technique resulting in a regularized matrix equation. Thus, numerical solution has a guaranteed accuracy (within the digital precision of a computer) even at sharp resonances, which is not the general case for the method of moments.

According to the numerical results, one can say that it is more preferable to choose a lossy-magnetic material for coating. Further, it is much better to make coating from inside to suppress the resonances when the interior resonance is the dominant feature in the backscattering characteristics. Otherwise, coating from outside can also be preferable to reduce the average level of the RCS off the resonant frequency. At direct on-aperture incidence, however, it is the inner magnetic coating which is definitely best at any frequency. Recalling that the same conclusion has been demonstrated in E-case [19], we emphasize that this is a far-from-obvious result. The fact that only a relatively thin lossy lining is needed to absorb the whole interior field in the cavity deserves a similar remark. Unlike the E-polarization case, there appears a low-frequency spike in the RCS for the H-polarization. This magnitude of this spike and the asymptotic values of natural frequencies of the cavity are given.

Although we have considered only circular, cylindrical cavities, the numerical data obtained can obviously bring a better understanding of the scattering behavior of loaded cavities of more general shape. It can also serve as reference data for checking numerical codes which use the method of moments or other numerical techniques [3]-[7].

\section{ACKNOWLEDGMENT}

The authors are indebted to the reviewers for helpful advice.

\section{REFERENCES}

[1] L. B. Felsen and G. Vecchi, "Wave scattering from slit coupled cylindrical cavities with interior loading: Part II-Resonant mode expansion," IEEE Trans. Antennas Propagat., vol 39, no. 8, pp. 1085-1097, Aug. 1991.

[2] K. Kobayashi and A. Sawai, "Plane wave diffraction by an open-ended parallel-plate waveguide cavity," J. Electromag. Waves Appl., vol. 6, pp. 475-512, 1992.

[3] J. R. Mautz and R. F. Harrington, "Electromagnetic penetration into a conducting circular cylinder through a narrow slot, TE case," $J$. Electromagn. Waves Appl., vol. 3, no. 4, pp. 307-336, 1989.

[4] A. El-Hajj, K. Y. Kabalan, and R. F. Harrington, "Characteristic modes of a slot in a conducting cylinder and their use for penetration and scattering, TE case," IEEE Trans. Antennas Propagat., vol 40, no. 2, pp. 156-161, Feb. 1992.

[5] T. M. Wang, A. Cuevas, and H. Ling, "RCS of a partially open rectangular box in the resonant region," IEEE Trans. Antennas Propagat., vol. 38, no. 9, Sept. 1990.

[6] P. M. Goggans and T. H. Shumpert, "Backscatter RCS for TE and TM excitations of dielectric-filled cavity backed apertures in 2-D bodies," IEEE Trans. Antennas Propagat., vol. 39, no. 8, pp. 1224-1227, Aug. 1991.

[7] J. A. Beren, "Diffraction of an H-polarized electromagnetic wave by a circular cylinder with an infinite axial slot," IEEE Trans. Antennas Propagat., vol AP-31, pp. 419-425, May 1983.

[8] A. Altıntaş, P. H. Pathak, and M. C. Liang, "A selective modal scheme for the analysis of EM coupling into or from large open-ended waveguides," IEEE Trans. Antennas Propagat., vol. AP-36, no. 1, pp. 84-96, Jan. 1988.

[9] A. Altıntaş, "Electromagnetic scattering from a class of open-ended waveguide discontinuities," Ph.D. dissertation, Dept. Electrical Eng., The Ohio State Univ., Columbus, OH, 1986.

[10] C. S. Lee and S. W. Lee, "RCS of a coated circular waveguide terminated by a perfect conductor," IEEE Trans. Antennas Propagat., vol. AP-35, no. 4, pp. 391-398, Apr. 1987.

[11] N. H. Myung, "A high frequency analysis of electromagnetic plane wave scattering by perfectly-conducting semi-infinite parallel-plate and rectangular waveguides with absorber coated inner walls," Ph.D. dissertation, Dept. Electrical Eng., The Ohio State Univ., Columbus, $\mathrm{OH}$, 1986 .

[12] H. Ling, R. C. Chou, and S. W. Lee, "Shooting and bouncing rays: Calculating the RCS of an arbitrarily shaped cavity," IEEE Trans. Antennas Propagat., vol. 37, no. 2, pp. 194-205, Feb. 1989.

[13] H. Shirai and L. B. Felsen, "Rays, modes and beams for plane wave coupling into a wide open-ended parallel-plane waveguide," Wave Motion, vol. 9, pp. 301-317, 1987.

[14] A. I. Nosich, "Green's function-dual series approach in wave scattering by combined resonant scatterers," in Analytical and Numerical Methods in EM Wave Theory, M. Hashimoto, M. Idemen and O.A. Tretyakov, Eds. Tokyo: Science House, 1993, pp. 419-469.

[15] "Electromagnetic characterization of unclosed circular cylindrical screens," Ph.D. dissertation, Dept. Radio Physics, Kharkov University, 1979 (in Russian)

[16] W. A. Johnson and R. W. Ziolkowski, "The scattering of an H-polarized plane wave from an axially slotted infinite cylinder: A dual series approach," Radio Sci. vol. 19, no. 1, pp. 275-291, Jan. 1984.

[17] R. W. Ziolkowski, "N-series problems and the coupling of electromagnetic waves to apertures: A Riemann-Hilbert approach," SIAM J. Math. Anal., vol. 16, no. 2, pp. 358-378, 1985.

[18] R. W . Ziolkowski and J. B. Grant, "Scattering from cavity-backed apertures: The generalized dual series solution of the concentrically loaded E-Pol slit cylinder problem," IEEE Trans. Antennas Propagat., vol. AP-35, no. 5, pp. 504-528, May 1987. (Note the error in RCS plots in Figs 19(a), 20(a), due to a problem with the computer code.)

[19] D. Colak, A. I. Nosich, and A. Altıntas, "Radar cross section study of cylindrical cavity-backed apertures with outer or inner material coating: 
The case of E-polarization," IEEE Trans. Antennas Propagat., vol. 41, pp. 1551-1559, Nov. 1993.

[20] M. Abramowitz and I. A. Stegun, Handbook of Mathematical Functions. New York: Dover, 1965.

[21] J. D. Jackson, Classical Electrodynamics, 2nd ed. New York: Wiley, 1975 , p. 453.

[22] R. F. Harrington, Time-Harmonic Electromagnetic Fields. New York: McGraw-Hill, 1961.

[23] A. I. Nosich and V. P. Shestopalov, "An electromagnetic analog of a Helmholtz resonator," Sov. Phys. Dokl., pp. 251-253, May 1977. (Note the missing factor " 2 " in the formula (6) for $k_{00}^{\prime}$, due to a misprint.)

[24] P. J. Clarricoats, P. E. Green, and A. A. Oliner, "Slot-mode propagation in rectangular waveguide," Electron. Lett., vol. 2, pp. 307-308, 1966.

[25] E. I. Veliev, A. I. Nosich, and V. P. Shestopalov, "Propagation of electromagnetic waves in a cylindrical waveguide with a longitudinal slit," Radio Eng. Electron. Physics, English translation, vol. 22, pp. 29-35, 1977.

[26] L. Rayleigh, "Theory of Helmholtz resonator," Proc. Royal Soc. London (A), vol. 92, pp. 265-275, 1915.

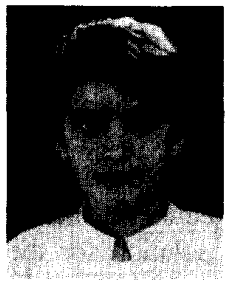

Dilek Colak (S'90) received the B.S. and M.S. degrees in electrical engineering from Bilkent University, Ankara, Turkey, in 1991 and 1993, respectively.

She is now a Graduate Research Associate at the ElectroScience Laboratory, The Ohio State University, working toward the Ph.D. degree. Her current research interests include analytical and numerical solution of electromagnetic scattering problems, radar cross section and antennas.

Ms. Colak was one of the recipients of the Young Scientist Award of the International Union of Radio Science (URSI) at the 24th General Assembly in 1993.

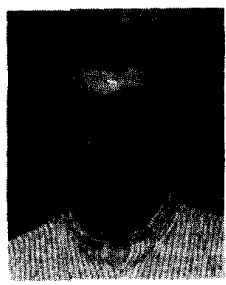

Alexander I. Nosich (M'94) graduated from the Radio Physics Department of Kharkov University in 1975. He received the Ph.D. and D.Sc. degrees in radio physics from Kharkov University, in 1979 and 1990 , respectively.

Since 1978, Dr. Nosich has been with the Electronics Department of the Institute of Radiophysics and Electronics (IRE) of the Ukranian Academy of Sciences, Kharkov, as a Junior and Senior Research Scientist. Currently, he is a Leading Scientist at the IRE. In 1992, he was with the Department of Electrical and Electronics Engineering of Bilkent University, Ankara, Turkey as a Visiting Professor through the Fellowship of Turkish Scientific and Technical Research Council (TÜBITAK). From 1992 to 1994, he was with the Department of Electrical Engineering and Computer Science of Kumamoto University, Japan as a Guest Scientist through the Fellowship of Matsumae International Foundation, and an Associate Professor. His current research interests are in free space and open waveguide scattering, complex modes behavior, radar cross section modifying, and antenna simulation.

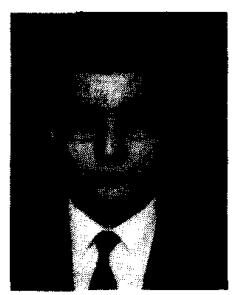

Ayhan Altuntas (S'82-M'86-SM'93) received the B.S. and M.S. degrees from the Middle East Tech nical University (METU), Ankara, Turkey, in 1979 and 1981, respectively, and the $\mathrm{Ph} . \mathrm{D}$. degree from The Ohio State University, Columbus, in 1986, all in electrical engineering.

From 1981 to 1987 , he was with the ElectroScience Laboratory, The Ohio State University. Afterward, he spent one year at the Optical Sciences Center of The Australian National University, Canberra, Australia. Currently, he is an Associate Professor with the Department of Electrical and Electronics Engineering, Bilkent University, Ankara, Turkey. His research interests are in electromagnetic radiation and scattering, microwaves, fiber optics, and integrated optics.

Dr. Altıntas is a member of Sigma Xi and Phi Kappa Phi. He was Chairman of IEEE Turkey Section for the term 1991-1993. He is the recipient of the ElectroScience Laboratory Outstanding Dissertation Award of 1986, IEEE 1991 Outstanding Student Branch Counselor Award, and the 1991 Research Award of Prof. Mustafa N. Parlar Foundation of METU. 